\title{
SPECTROSCOPIC AND OPTICALLY DETECTED MAGNETIC RESONANCE STUDIES OF PO- IN POTASSIUM CHLORIDE. II. MAGNETIC RESONANCE ANALYSIS
}

\author{
S.J. HUNTER, K.W. HIPPS and A.H. FRANCIS \\ Department of Chemistry, Unizersity of Michigan. Ann Arbor, Michigan 48109. US.A
}

Received 29 November 1978

\begin{abstract}
The optically detected magnetic resonance (ODMR) spectrum of phosphorus activated potassium chloride crystals obtained at $2 \mathrm{~K}$ in zero and weak applied external magnetic fields is reported and analyzed. A theoretical fit to the observed field and angular dependence of the ODMR spectrum is obtained using a phenomenological spin hamiltonian, assuming the center to be the paramagnetic photoexcited ${ }^{3} \mathrm{~B}_{1}$ state of the $\mathrm{C}_{2 v}$ ion $\mathrm{PO}_{2}^{-}$. The components of the phosphorus nuclear hyperfine structure tensor are obtained from the theoretical analysis and compared with those of other $\mathrm{AB}_{2}$ ions and radicals. Optical polarization measurements and weak ficld ODMR spectra are used to determine the orientation of the $\mathrm{PO}_{2}^{-}$chemical impurity center in the cubic $\mathrm{KCl}$ lattice and the orientation is compared with those of other isostructural impurities in $\mathrm{KCl}$. The $\mathrm{PO}_{2}^{-}$chemical impurity center exhibits a dramatic photo-orientation effect at $2 \mathrm{~K}$ and is shown to be in thermal equilibrium between twelve crystallographically equvalent orientations at temperatures above $45 \mathrm{~K}$.
\end{abstract}

\section{Introduction}

In a previous article [1] we reported the preliminary results of an optically detected magnetic resonance (ODMR) study of phosphorus activated potassium chloride crystals. It is the purpose of the present article to present the full details of that investigation. The spectroscopic results have been reported in part $\mathbf{I}$ of this work, ${ }^{\dagger}$ and only such aspects of these as are required for an interpretation of the magnetic resonance data will be repeated here.

This investigation was undertaken to further establish the chemical identity and crystallographic orientation of the center produced in alkali halide lattices by heating in phosphorus vapors. This center is of particular spectroscopic interest due to the details of the narrow line absorption and emission spectrum and also for several aspects of the strong electron-phonon coupling evident in the spectrum.

' Chem. Phys. 39 (1979) 209.

\section{Experiments}

The method of preparation of phosphorus activated potassium chloride has been described previously [2-4]. The optical arrangement and cryogenic apparatus used in the magnetic resonance experiments were the same, in large part, as those empluyed in the work described in part I. Only the aspects of the experimental apparatus which differ significantly from those previously described will be mentioned here. In the measurements to be described, the total sample emission was monitored using a sharp cut Corning 3-75 glass filter to separate the sample emission from the scattered excitation radiation which had been passed by a $\mathrm{Ni} / \mathrm{CoSO}_{4}$ solution filter and a Corning 7-54 glass filter. In this manner, a significantly better signal/ noise ratio was obtained than if a dispersing monochromator was used. Additionally, a 3M Co. PL-40 polarizing filter was inserted as a polarizer between the excitation source and the sample, and as an analyzer between the sample and the RCA 1 P28 photomultiplier used to detect phosphorescence. 
Crystals were cleaved parallel to the principal cleavage planes of the cubic lattice $\{100\}$, to approximate dimensions $2 \mathrm{~mm} \times 2 \mathrm{~mm} \times 3 \mathrm{~mm}$ and mounted in a slow wave microwave helix attached to the end of a three foot length of semirigid cryogenic coaxial cable. The rigid coaxial cable served both to support and orient the crystal in the field of a 1 inch diameter American Magnetics superconducting solenoid in the Helmholtz arrangement. Field homogeneity was about $0.01 \%$ over the volume of the crystal sample when placed between the Helmholtz windings on the solenoid axis. The split solenoid arrangement permitted large aperature optical excitation of the sample phosphorescence at right angles to the direction of observation.

The orientation of the sample crystal with respect to the direction of the applied magnetic field could be controlled by rotation of the entire coaxial cable and helix assembly within the solenoid about one axis only. The accuracy of the angular measurements was limited principally by the mechanical linkage employed to transmit motion from the top of the cryostat to the liquid helium bath and was on the order of $\pm 2^{\circ}$.

The superconducting solenoid was powered by a current regulated Sorensen SRL 10-50 power supply and field strengths were obtained from the magnet current using the manufacturer supplied field to current ratio. The estimated accuracy of the absolute field strength was $\pm 1 \%$ -

\section{Results}

Two types of optically detected magnetic resonance measurements were conducted. In the first, the Zeeman splitting of the zero-field resonance transitions was determined as a function of applied field strength for several orientations of the field using unpolarized excitation and monitoring the total sample emission. Results typical of this type of measurement are shown in fig. 1 for the 4580 $\mathrm{MHz}$ zero-field resonance with the magnetic field parallel to the direction of observation and in fig. 2 for both the $2930 \mathrm{MHz}$ and $4580 \mathrm{MHz}$ zero-field resonances for the field parallel to the direction of excitation. Only the behavior of the four most intense Zeeman components is illustrated in fig. 2.

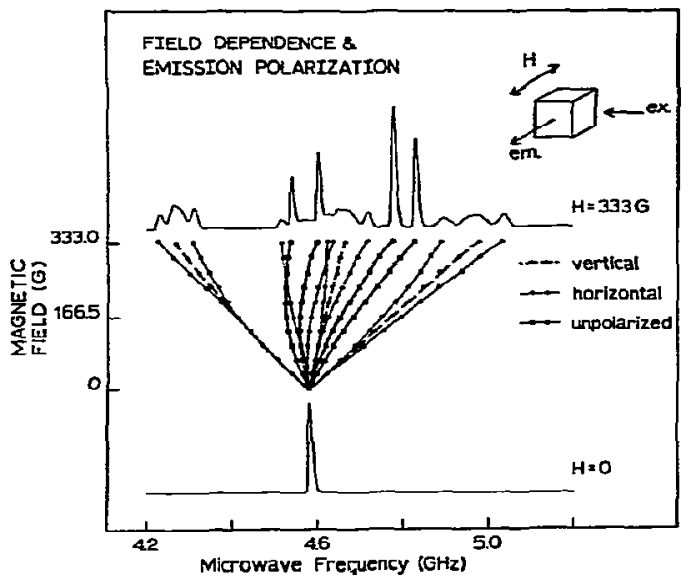

Fig. 1. The weak field splitting pattern of the $4580 \mathrm{MHz}$ zero-field ODMR obtained with the magnetic field parallel to the direction of observation of phosphorescence. The individual components are optically polarized in emission as indicated in the diagram.

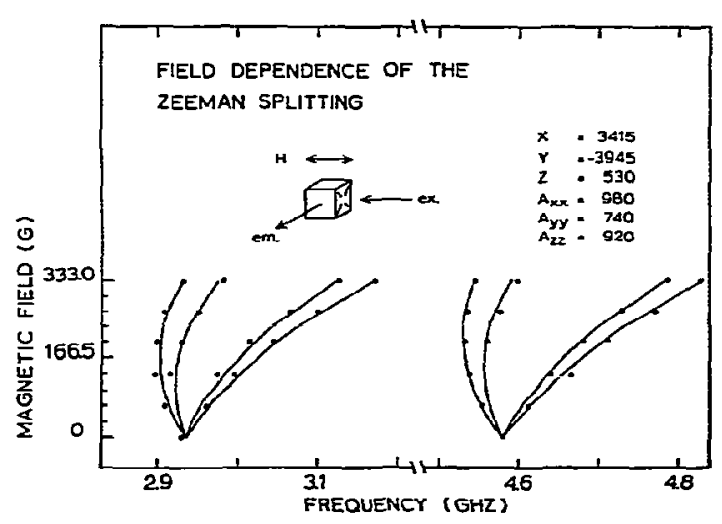

Fig. 2. The weak field splitting pattern of the $2930 \mathrm{MHz}$ and $4580 \mathrm{MHz}$ zero-field ODMR spectra obtained with the magnetic field parallel to the direction of excitation of phosphorescence. The solid lines indicate the theoretical fit abtained using the spin hamiltonian of eq. (1).

A linear dependence of the splitting upon applied field is observed at low field ( $<50 \mathrm{G}$ ), while at high field the splitting varies approximately quadratically.

The optical polarization of the individual Zeeman field components could be investigated by inserting a polarizer between the excitation source and the sample and an analyzer between the sample and the 


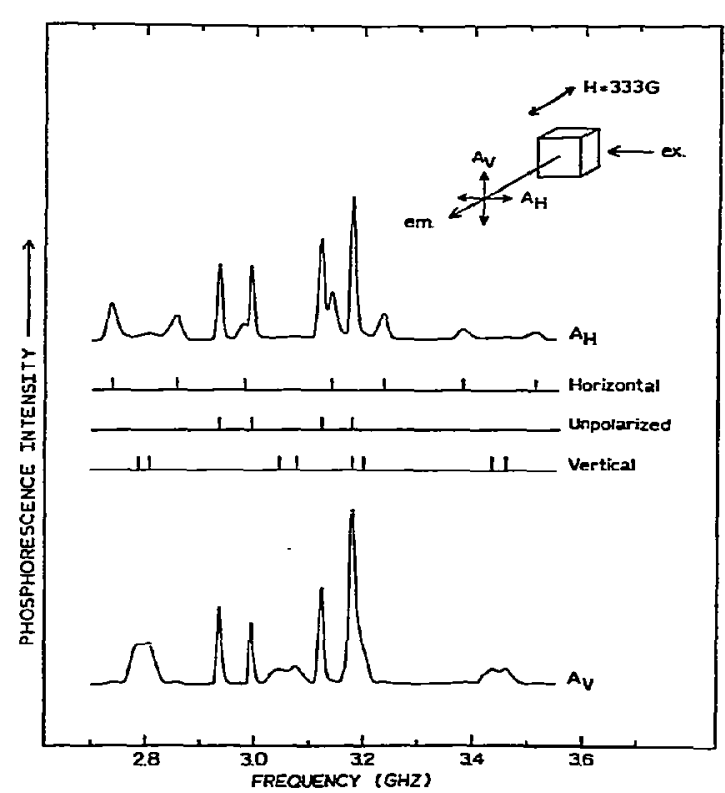

Fig. 3. Optical polarization in emission of the individual components of the weak field splitting pattern of the 2930 $\mathrm{MHz}$ zero-field resonance.

photomultiplier used to monitor total emission intensity. The polarization of the total Zeeman splitting pattern is illustrated in fig. 3 .

The second type of measurement involved the determination of the Zeeman splitting pattern as a function of the direction of the applied magnetic field. Experiments were conducted in which the magnetic field was rotated through the $\{100\}$ and $\{110\}$ planes. The angular dependence of the Zeeman field splitting pattern for the $2930 \mathrm{MHz}$ zero-field resonance as the applied field was rotated through the $\{100\}$ plane is illustrated in fig. 4 . The solid circles represent the data points and the continuous curves the theoretical fit obtained from the spin Hamiltonian of eq. (1) below. In order to simplify the diagram, the data has been separated vertically into two sets. The experimental measurements were performed by rotating the crystal, while maintaining the direction of the field fixed with respect to the direction of excitation.

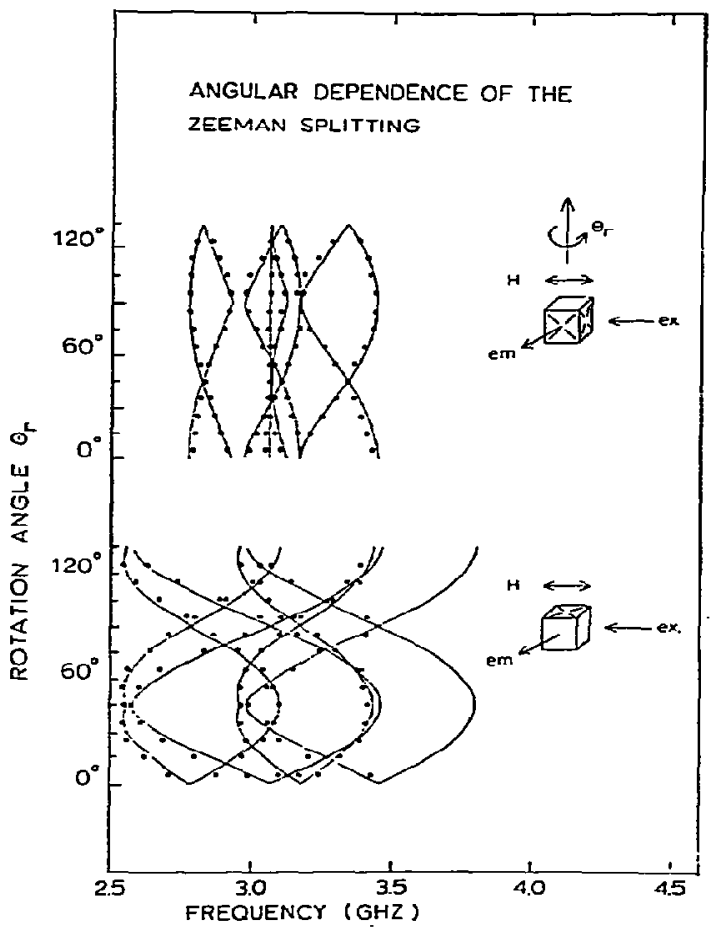

Fig. 4. Angular dependence of the total splitting pattern of the $2930 \mathrm{MHz}$ zero-field resonance for a field of $333 \mathrm{G}$ parallel to the direction of excitation. Rotation is about the $[100]$ axis which is perpendicular to both the magnetic field and the directions of excitation and observation.

\section{Discussion}

\subsection{The spin-hamiltosian}

It will be shown that our ODMR spectra can be described by the following spin hamiltonian [5]:

$\boldsymbol{H}=\boldsymbol{B} \boldsymbol{S} \cdot \hat{\mathbf{g}} \cdot \boldsymbol{H}+\boldsymbol{S} \cdot \hat{\mathbf{D}} \cdot \boldsymbol{S}+\sum_{i} I_{i} \cdot \overline{\mathbf{A}} \cdot \boldsymbol{S}$

where $\beta$ is the Bohr magneton, $H$ is the applied magnetic field, $S$ and $\boldsymbol{I}$ are the electron and nuclear spin operators: $\hat{\mathbf{g}}, \hat{\mathbf{D}}$ and $\hat{\mathbf{A}}$ are the fine structure tensor, the electron spin-spin dipolar coupling tensor and the electron-nuclear hyperfine coupling tensor, respectively. Nuclear Zeeman terms have been omitted from the hamiltonian since they are not appreciable at the low $(<1 \mathrm{kG})$ magnetic fields employed. Additionally, since it is anticipated that 
the electron density is highly localized on the impurity center, the summation does not extend over lattice nuclear spins.

The zero order wavefunctions for electron and phosphorus nuclear spin were the six functions $\left|M_{S}, M_{I}\right\rangle$, with $S=1$ and $I=1 / 2$. The resulting matrix elements of the hamiltonian (1) were obtained in terms of the spherical polar coordinate angles $\phi$ and $\theta$, which define the direction of the applied magnetic field with respect to the molecular symmetry axes $X, Y$ and $Z$ defined as in part $I$.

At low applied magnetic field $(H<1 \mathrm{kG})$ the electronic Zeeman term is comparable to the spindipolar term. Additionally, since the phosphorus isotropic hyperfine term in eq. (1) may also be of magnitude comparable to the spin dipolar and Zeeman terms, fits to the experimental data were obtained by diagonalization of the full $6 \times 6$ determinant for various choices of the angular variables $\phi$ and $\theta$. For the purpose of the analysis of the hyperfine structure, the $g$-tensor anisotropy was neglected. This is justified by the fact that only low fields were employed and the anisotropy was small compared to the hyperfine field. The axes of the $g$-tensor, fine structure and hyperfine tensor must all be parallel to the molecular symmetry axes of the $\mathrm{C}_{2 v}$ ion $\mathrm{PO}_{2}^{-}$.

Using the spin hamiltonian of eq. (1), the Zeeman field splitting pattern shown in fig. 2 could be fit to within experimental error in angular alignments. Moreover, the full angular dependence of the Zeeman field splitting could also be reproduced in all major features (see fig. 4).

In all crystals studied the observed ODMR peaks could be ascribed to one of twelve crystallographically equivalent $\mathrm{PO}_{2}^{-}$orientations, and it is concluded that in low concentration in $\mathrm{KCl}$ this orientation is present exclusively. The final values of the zero-field and phosphorus hyperfine coupling constants are given in fig. 2.

Although angular measurements could not be made to an accuracy of better than $\pm 2^{\circ}$, this provided sufficient accuracy to ascertain the correctness of the spin hamiltonian and establish the orientation of the centers. However, it was not profitable to attempt further refinement of the theoretical fit in an attempt to determine the $g$-tensor anisotropy.
Table 1

Nuclear hyperfine structure parameters (in $\mathrm{MHz}$ ) for some selected $\mathrm{AB}_{2}$ type radicals

\begin{tabular}{lcccccc}
\hline $\begin{array}{l}\text { Molecule } \\
\text { or ion }\end{array}$ & $\begin{array}{l}\text { Orbital } \\
\text { configu- } \\
\text { ration }\end{array}$ & $B_{x x}$ & $B_{y y}$ & $B_{z z}$ & $A_{0}$ \\
\hline $\mathrm{C}^{13} \mathrm{O}_{2}^{-}[6]$ & $\sigma_{3}^{1}$ & -32.0 & -46.0 & 78 & 469 \\
$\mathrm{NO}_{2}[7]$ & $\sigma_{3}^{1}$ & -14.8 & -22.3 & 37 & 153 \\
$\mathrm{PO}_{2}^{-}$ & $\sigma_{3}^{1} \pi_{2}^{1}$ & 100 & -140 & 40 & 880 \\
$\mathrm{NO}_{2}^{-}[8]$ & $\sigma_{3}^{1} \pi_{2}^{1}$ & 28 & -27 & - & 1 & 100 \\
$\mathrm{SO}_{2}^{-}$ & {$[9]$} & $\pi_{2}^{1}$ & 113 & -54 & -57 & 35 \\
$\mathrm{NO}_{2}^{2-}[10,11]$ & $\pi_{2}^{2}$ & 46.5 & -26 & -20.5 & 40.1 \\
$\mathrm{ClO}_{2}^{2}[12,13]$ & $\pi_{2}^{1}$ & 161.5 & -86.3 & -75.1 & 43.2 \\
$\mathrm{SeO}_{2}^{-}[14]$ & $\pi_{2}^{1}$ & +277 & -124 & -153 & 393
\end{tabular}

\subsection{Hyperfine analysis}

The principal values of the hyperfine structure tensor $\left(A_{x x}, A_{y y}\right.$ and $\left.A_{z z}\right)$ of an $\mathrm{AB}_{2}$ type molecule or ion in which only the central atom possesses a nuclear spin arc determined by the elcetron spin distribution about the central nucleus. The orbital configuration of the excited ${ }^{3} \mathrm{~B}_{1}$ state of $\mathrm{PO}_{2}^{-}$is $\sigma_{3}^{1} \pi_{2}^{1}$, where we have employed the symbolism used in part $I$. Therefore, the unpaired spin distribution is described by two molecular orbitals, one of which $\left(\pi_{2}\right)$ has a node at the molecular plane and, therefore, no appreciable electron density at the phosphorus nucleus. The other molecular orbital $\left(\sigma_{3}\right)$ lies in the plane of the molecule and has appreciable electron density at phosphorus. The electron distribution of $\sigma_{3}$ about the phosphorus nucleus may be approximately described by an $\mathrm{sp}^{2}$ hybridization of the phosphorus valence orbitals.

The hyperfine interaction may be divided into an isotropic part due to the spherically symmetric part of the electron distribution and specified by a scalar $\left(A_{0}\right)$ and an anisotropic part specified by a traceless tensor with components $B_{x x}, B_{y y}$, and $B_{z z}$ - Thus:

$$
\begin{aligned}
& 0=B_{x x}+B_{y y}+B_{z z}, \\
& \left|A_{x x}\right|=\left|A_{0}+B_{x x}\right|, \\
& \left|A_{y y}\right|=\left|A_{0}+B_{y y}\right|, \\
& \left|A_{z z}\right|=\left|A_{0}+B_{z z}\right| .
\end{aligned}
$$

In the present instance, the isotropic hyperfine interaction is dominated by the Fermi contact 
interaction due to s-electron density at the nucleus and given by:

$A_{0}=\frac{4}{3} h \gamma_{\mathrm{e}} \gamma_{\mathrm{A}}\left|\psi_{\mathrm{S}}(0)\right|^{2}$,

where $\gamma_{\mathrm{A}}$ is the gyromagnetic ratio of the central nucleus and $\left|\psi_{s}(0)\right|^{2}$ is the s-electron density at the central nucleus. The anisotropic hyperfine interaction is due to the p-electron distribution on phosphorus. Referring to the molecular orbitals $\pi_{2}$ and $\sigma_{3}$, it is apparent that to good approximation $\pi_{2}$ contributes to the anisotropic hyperfine interaction only, while $\sigma_{3}$ contributes to both the isotropic and anisotropic interactions.

The relative signs of the principal values of the hyperfine structure tensor cannot, in general, be determined from the data obtained in the ODMR spectra, therefore, three possible choices of $A_{0}$ and $B_{i i}$ are compatible with eqs. (2). However, the large s-electron density at the phosphorus nucleus contributed by the $\sigma_{3}$ orbital, combined with the very large isotropic hyperfine interaction of phosphorus $(10,178 \mathrm{MHz})$ assure that the components of the hyperfine interaction will be dominated by the Fermi contact (isotropic) interaction and, therefore, all be positive. With this assumption it is possible to determine the values of the principal components of the anisotropic interaction as well as the magnitude of the isotropic interaction from eqs. (2a)-(2d):

$A_{0}=880 \mathrm{MHz}, B_{x x}=100 \mathrm{MHz}, B_{y y}=-140 \mathrm{MHz}$, $B_{z z}=40 \mathrm{MHz}$.

The hyperfine coupling parameters for a small number of $\mathrm{AB}_{2}$ molecules and ions are collected in table 1 for comparison with the results obtained for $\mathrm{PO}_{2}^{-}$[15]. Included in the table are ions with 19 and 17 valence electrons, one more and one less, respectively, than the 18 valence electrons of $\mathrm{PO}_{2}^{-}$. Thus, with the exception of $\mathrm{NO}_{2}^{-}$with 18 valence electrons, the species tabulated are radicals or radical ions in their electronic ground states and the data shown has been obtained from conventional ground state ESR measurements.

The hyperfine coupling parameters obtained for $\mathrm{PO}_{2}^{-}$may be shown to be in quantitative agreement with the hyperfine parameters of other isostructural and isoelectronic ions. The 17-electron radical $\mathrm{NO}_{2}$ and the radical ion $\mathrm{CO}_{2}^{-}$both have the unpaired electron in the $\sigma_{3}$ molecular orbital. The electron distribution in $\sigma_{3}$ at the central nucleus may be approximately described by an $\mathbf{s p}^{2}$ atomic hybrid orbital and, consistent with this distribution, the anisotropic hyperfine interaction is greatest along the two-fold $(Z)$ molecular axis. The relatively large isotropic hyperfine interaction results from the considerable s-orbital character of $\sigma_{3}$ at the central nucleus.

A rough estimate of the isotropic hyperfine interaction for $\mathrm{PO}_{2}^{-}$may be obtained by scaling the isotropic hyperfine interaction of $\mathrm{NO}_{2}$ by the ratio $\gamma_{P} / \gamma_{N}=5.61$ where $\gamma_{P}$ and $\gamma_{N}$ are the gyromagnetic ratios of phosphorus and nitrogen, respectively. In this manner, the isotropic hyperfine interaction due to the $\sigma_{3}$ spin distribution is estimated to be $860 \mathrm{MHz}$, which is in excellent agreement with the experimentally observed value of $880 \mathrm{MHz}$.

The hyperfine interaction due to the $\pi_{2}$ spin distribution may be estimated by consideration of the nineteen electron radical inn $\mathrm{SO}_{\bar{z}}^{-}$, which has one unpaired spin in the $\pi_{2}$ orbital. The spin distribution at the central nucleus is approximately described by a $p_{x}$ atomic orbital, and the anisotropic hyperfine interaction is greatest along the $X$ axis, with approximate axial symmetry in the molecular plane. Only a very small admixture of s-orbital character is necessary to account for the $\sigma_{3}$ distribution in 19-electron radicals and radical ions. The amount of s-orbital character necessary to account for the observed isotropic hyperfine interaction may be estimated using eq. (3) and the values of $\left|\psi_{s}(\theta)\right|^{2}$ for carbon and nitrogen obtained from selfconsistent-field calculations. A pure $3 \mathrm{~s}$ electron on sulfur has an isotropic hyperfine interaction of $2715 \mathrm{MHz}$. Therefore, the s-electron character at the central nucleus is of the order of $1 \%$ and the electron distribution of $\pi_{2}$ at the central nucleus is accurately described by an atomic $p_{x}$ orbital. We conclude that there is negligible contribution to the isotropic hyperfine interaction by the $\pi_{2}$ distribution in $\mathrm{PO}_{2}^{-}$.

\subsection{Molecular orientation}

The $\mathrm{C}_{2 \mathrm{v}}$ symmetry of the $\mathrm{PO}_{2}^{-}$ion requires that the molecular symmetry axes and the axes of the spin hamiltonian be parallel. Since the orientation of 
the $\mathrm{PO}_{2}^{-}$center is not initially known, the correspondence between the molecule fixed symmetry axes and the magnetic axes must be established by a series of magnetophotoselection experiments and theoretical argument. It has been previously argued (part I) that the direction of the electric dipole transition moment corresponding to the excitation ${ }^{1} \mathrm{~B}_{1} \leftarrow{ }^{1} \mathrm{~A}_{1}$ is parallel to the molecular $X$ axis. Additionally, the phosphorescence due to the Iransition ${ }^{3} B_{1}\left(\tau_{z}\right) \rightarrow{ }^{1} A_{1}$ is predominantly $Y$ axis polarized. Thus, the absorption (excitation) and emission polarizations together define the $X Y$ plane of the ion.

When an external magnetic field is applied, the individual zero-field resonance lines are split into as many as forty-eight components for an arbitrary field orientation, each with a characteristic optical polarization behaviour. For example, the $4580 \mathrm{MHz}$ resonance is split as illustrated in fig. 1 by a $333 \mathrm{G}$ field applied parallel to both [100] and the direction of observation of the optical emission. The complex multi-line pattern is composed of separate quartets. each member of which is identical in its optical excitation and emission polarization characteristics. The polarization properties for this orientation of the sample may be distinguished as either exclusively vertical, exclusively horizonial or unpolarized. The most easily distinguished quartet in fig. 1 is that composed of the four most intense bands of the multi-line pattern. This set of lines which is $100 \%$ vertically polarized in excitation is unpolarized in emission as shown in fig. 3. Therefore, this quartet belongs to a set of ions whose $X Y$ plane is normal to both the direction of observation and the external field. Moreover, since all orientations generated by the point operations of the cubic space group are equally probable, the $X$ and $Y$ molecular axes must lie parallel to $[110]$ tetragonal axes. Illustrated in fig. 5 are the twelve crystallographically equivalent orientations of the $\mathrm{PO}_{2}^{-}$ion in the cubic $\mathrm{KCl}$ lattice. The molecular orientation is specified by a vector representing the direction of the absorption $(X)$ or the emission ( $Y$ ) transition dipole moment. The orientational degeneracy is sufficiently high that neither optical photo-selection methods nor low field ODMR uniquely determine the orientation of the ion. However, a combination of the external magnetic field and optical-photoselection reduced the orientational degeneracy sufficiently to permit unambiguous determination of the orientation of the $\mathrm{PO}_{2}^{-}$center. The several experimental orientations of the crystallographic axes with respect to the external magnetic field and the directions of excitation and observation are illustrated in ligs. 5a, $5 b$ and $5 c$. For each orientation the twelve crystallographically equivalent orientations are separated into smaller groups of optically and magnetically equivalent ions. The individual sets of magnetically equivalent molecules in each figure can be distinguished in the optically detected magnetic resonance spectrum and the polarization properties of each set of lines investigated as a function of the polarization of the incident and emitted radiation. A series of photo-selected emission spectra for the different orientations of the cubic crystallographic axis system with respect to an applied magnetic field produced the results summarized in fig. 5 .

The interpretation of the polarization intensities is complicated by the fact that the $\mathrm{PO}_{2}^{-}$center undergoes rapid photo-reorientation which interconverts optically inequivalent sets of ions. This behavior required making time resolved polarization measurements subsequent to equilibration of the sample. The details of the photo-reorientation process will be discussed in part III of this work.

In order to correlate the magnetic and symmetry axes of the ion, the emission of the four crystallographically and magnetically equivalent ions whose molecular $X Y$ planes were normal to both the applied magnetic field and the direction of illumination was selected for study (set I of fig. 5a). This was accomplished with horizontally polarized excitation and a vertical analyzer. All other orientations of the ions are either not optically excited or do not emit vertically polarized radiation and, therefore, cannot be observed. Under these conditions the magnetic field is perpendicular to the molecular $X Y$ plane of the photo-selected set and, therefore, parallel to the molecular $Z\left(C_{2}\right)$ axis. The weak field splitting pattern for this set of ions is shown in fig. 2 . The observation that all Zeeman components increase in frequency with increasing field is sufficient to establish $\tau_{z}$ as the central spin-orbital state, in agreement with previous conclusions based on the PMDR spectra. The spin hamiltonian of eq. (1) was then used to obtain the solid line theoretical fit to 


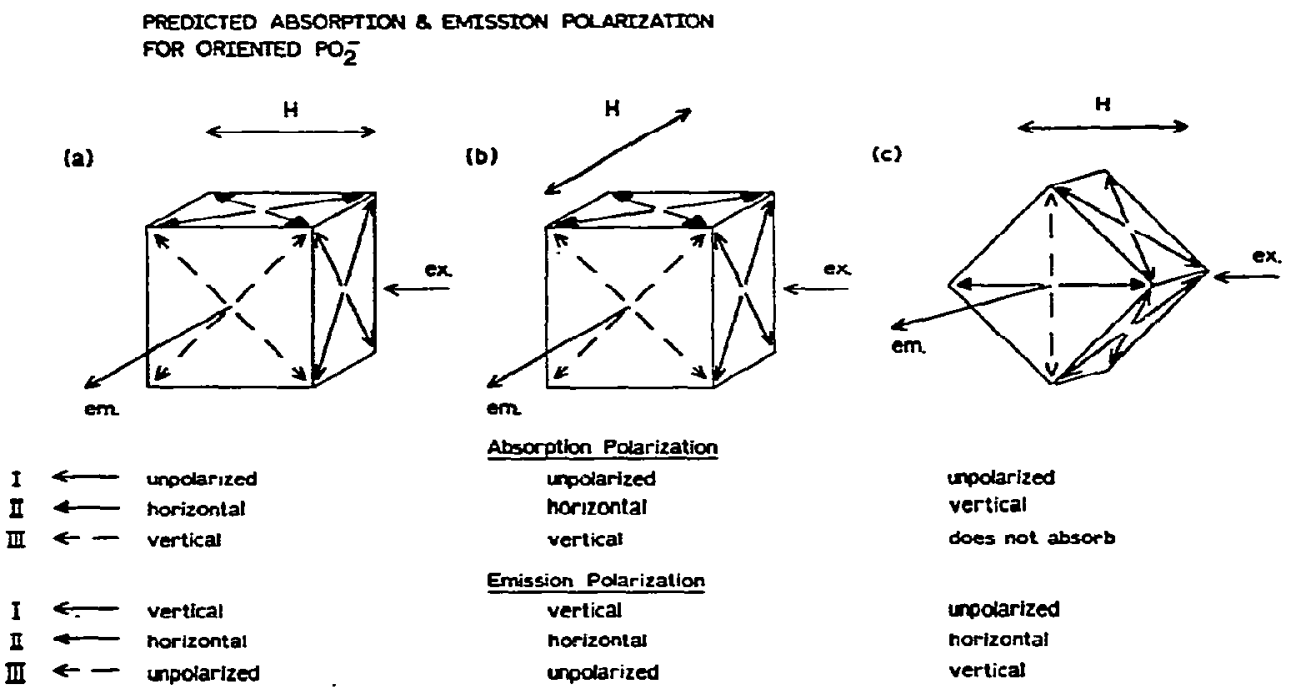

Fig. 5. The twelve crystallographically equivalent orientations of the impurity ion $Y$ axis, indicated by vectors in $\{100\}$ faces. The absorption and emission polarizations of the magnetically inequivalent sets are illustrated below each experimental orientation for which data was obtained.

the Zeeman splitting pattern illustrated in fig. 2 with $H$ parallel to $\mathcal{Z}$.

To establish the correlation of the magnetic and symmetry $X$ and $Y$ axes, the field and polarization orientation illustrated in fig. $5 c$ was employed. The final fine structure parameters and phosphorus hyperfine coupling constants given in fig. 2 were used to obtain the solid line theoretical fit to the angular dependence of the Zeeman field splitting of all ion orientations which is shown in fig. 4. The agreement is sufficiently good to establish the correctness of the model for the phosphorus center.

\section{Conclusion}

A review of the pertinent literature suggests that the primary consideration governing impurity center formation in alkali halides is charge compensation. The majority of triatomic singly charged anion centers in alkali halides occupy a halide ion vacancy, and we shall assume that this is also the case for $\mathrm{PO}_{\mathbf{2}}^{-}$- It is not possible to establish this unambiguously, however, from the magnetic resonance measurements, since no attempt was made to observe and analyze either the potassium or the chloride ion nuclear hyperfine structure to determine the symmetry and character of the surrounding nuclear spins. The orientation of the symmetry axes established for triplet $\mathrm{PO}_{2}^{-}$in $\mathrm{KCl}$ is illustrated in figs. 6 and 7. The dimensions of the $\mathrm{PO}_{2}^{-}$ion have been estimated from standard $\mathrm{P}-\mathrm{O}$ double bond lengths [16] and the bond angle determined from the valence force calculation in part I. In fig. 7 the chloride ion vacancy volume is indicated by the spherical cavity into which the $\mathrm{PO}_{2}^{-}$has been drawn.

Since phosphorus is considerably less electronegative than oxygen, the ion possesses a substantial dipole moment with the oxygens negative. In the orientation pictured the oxygen atoms are directed toward the positive potassium centers. The twelve equivalent orientations of the $\mathrm{PO}_{2}^{-}$ion are obtained by directing the two-fold axis toward the six potassium centers illustrated in fig. 7 and rotating $90^{\circ}$ about the molecular $Z$ axis. Evidently the potential barrier for reorientation of the $Z$ molecular axis is sufficiently low that rapid interconversion of the twelve equivalent orientations occurs above about $45 \mathrm{~K}$. We have observed the thermal reorientation by follow- 


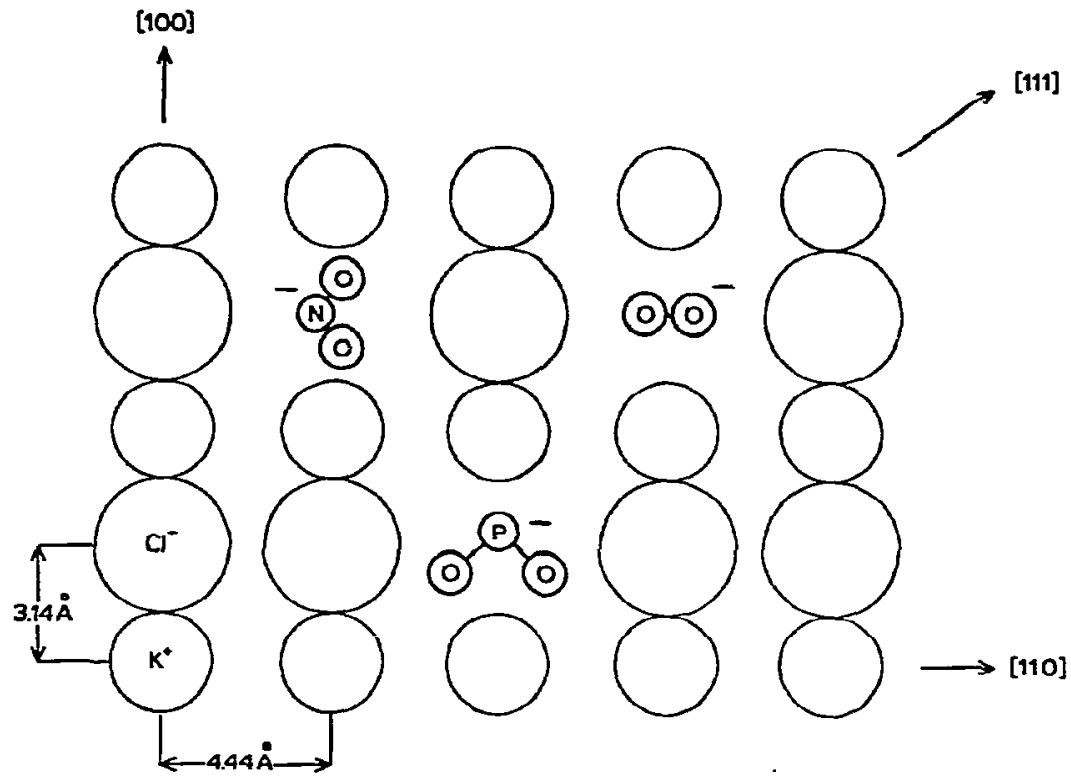

Fig. 6. The orientation established for the impurity ion $\mathrm{PO}_{2}^{-}$and several related ions in the $\{110\}$ plane of $\mathrm{KCl}$.

ing the loss of emission polarization from a photoaligned sample. The effect of the rapid interconversion may also be observable in the IR or Raman

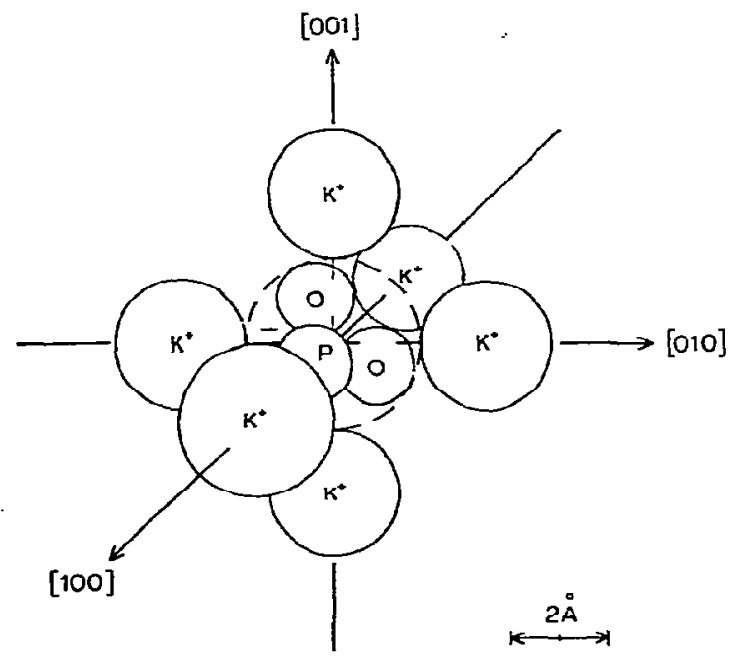

Fig. 7. One of the twelve crystallographically equivalent orientations of the impurity ion in a chloride ion vacancy. spectra of the center at elevated temperatures $[17,18]$.

Both the orientation and dynamical behavior of the ion $\mathrm{PO}_{2}^{-}$in $\mathrm{KCl}$ are similar to those determined for the radical ions $\mathrm{SO}_{2}^{-}$and $\mathrm{SeO}_{2}^{-}$by conventional ground state ESR measurements [9]. The sulfur and selenium triatomic ions are formed in situ in alkali halides when crystals containing $S_{3}^{-}$or $\mathrm{Se}_{3}^{-}$centers are heated in air. Rapid reorientation at elevated temperatures of the $\mathrm{SO}_{2}^{-}$and $\mathrm{ScO}_{2}^{-}$ions in chioridc ion vacancies leads to a dramatic narrowing of the ESR linewidths, due to averaging of the ${ }^{33} \mathrm{~S}$ and ${ }^{77} \mathrm{Se}$ anisotropic nuclear hyperfine structure.

The nitrite ion, $\mathrm{NO}_{2}^{-}$, has been extensively studied [17-22] as a chemical impurity center in $\mathrm{KCl}$ where it is known to occupy a chloride ion vacancy. Like $\mathrm{PO}_{2}^{-}, \mathrm{SO}_{2}^{-}$and $\mathrm{SeO}_{2}^{-}$, the molecular plane of $\mathrm{NO}_{2}^{-}$coincides with $\{110\}$ crystal planes. However, unlike the first three.ions, the two-fold axis of $\mathrm{NO}_{2}^{-}$is directed along [110] rather than [100] crystal directíons. The unique orientation and smaller size of the nitrite center evidently allows nearly free rotation at low temperatures, suggesting a significantly lower barrier to reorientation than that for the sulfur, selenium and phosphorus centers. 


\section{References}

[1] S.J. Hunter, K.W. Hipps and A.H. Francis, Chem. Phys. Letters 51 (1977) 287.

[2] B.T. Plachenov, V.P. Avdonin, G.A. Mikhalchenko and V.M. Smagin, Izv. Akad. Nauk SSSR Bull. Phys. Ser. 30 (1966) 1474.

[3] B.T. Plachenov, V.P. Avdonin, V. Kapishovskii and G.A. Mikhal'chenko, lzv. Akad. Nauk SSSR Bull. Phys. Ser. 31 (1967) 2067.

[4] R.A. Avarmaa, Opt. Spectry. 32 (1972) 514.

[5] J.E. Wertz and J.R. Bolton, Electron spin resonance: Elementary theory and practical applications (McGraw-Hill, New York 1972).

[6] D.W. Ovenall and D.H. Whiffen, Mol. Phys. 4 (1961) 135.

[7] H. Zeldes and R. Livingston, J. Chem. Phys. 35(1961) 563.

[8] J.U. von Schutz and W. Dietrich, Chem. Phys. Letteis 51 (1977) 418.

[9] J. Schneider, B. Dischler and A. Rauber, Phys. Stat. Sol. 13 (1966) 141.

[10] C. Jaccard. Phys. Rev. 124 (1961) 60.
[11] P.W. Atkins and M.C.R. Symons, J. Chem. Soc. (1962) 4794.

[12] T. Cole, Proc. Natl. Acad. Sci. US, 46 (1960) 506.

[13] P.W. Atkins, J.A. Brivati. N. Keen. M.C.R. Symons and P.A. Trevalion, J. Chem. Soc. (1962) 4785.

[14] Y. Kurita and W. Gordy, J. Chem. Phys. 34 (1961) 1285.

[15] P.W. Atkins and M.C.R. Symons, The structure of inorganic radicals (Elsevier, Amsterdam, 1967).

[16] R.C. Weast, Handbook of chemistry and physics (The Chemical Rubber Co., Ohio, 1971).

[17] A.R. Evans and D.B. Fitchen, Phys. Rev. B2 (1970) 1074.

[18] V. Narayanamurti, W.D. Seward and R.O. Pohl, Phys. Rev. 148 (1966) 481.

[19] R. Avarmaa and L.A. Rebane, Phys. Stat. Sol. 35 (1969) 107

[20] L.A. Rebane, G.S. Zavt, and K.K. Haller, Phys. Stat. Sol. 81 (1977) 57.

[21] R. Bonn, R. Metselaar and J. van der Elsken, J. Chem. Phys. 46 (1967) 1988.

[22] T.J. Haldre, L.A. Rebane, A.V. Liapzev and A.A. Kiselev, Phys. Stat. Sol. B70 (1975) 359. 